\title{
EFECTO AGUDO DEL ENTRENAMIENTO MÁXIMO DE FLEXIBILIDAD SOBRE LA FUERZA MUSCULAR Y LOS NIVELES DE HIDROXIPROLINA URINARIA EN JÓVENES ADULTOS ACUTE EFFECT OF MAXIMAL STRETCHING TRAINING ON MUSCULAR STRENGTH AND URINARY HYDROXYPROLINE LEVELS OF YOUNG ADULTS
}

\author{
Almeida, Olavo 1,2, Mello, Danielli 1,3, Vale, Rodrigo 1, Rosa, Guilherme 1, Sampaio, Adriane 4, Daoud, Rejane 1, \\ Luque, Antonio 5 \& Dantas, Estelio 1.
}

\author{
1 Laboratório de Biociências da Motricidade Humana - LABIMH/UNIRIO. \\ 2 Faculdade Santa Rita (FASAR) - Conselheiro Lafaiete / MG / Brasil. \\ 3 Escola de Educação Física do Exército - EsEFEx/EB. Brasil. \\ 4 Escola Preparatória de Cadetes do Ar - Barbacena / MG / Brasil. \\ 5 Laboratorio de Fisiología del Ejercicio - Universidad Católica de Murcia/España.
}

ALMEIDA O.; MELLO D.; VALE R.; ROSA G.; SAMPAIO A.; DAOUD R.; LUQUE A. \& DANTAS E. Efecto agudo del entrenamiento máximo de flexibilidad sobre la fuerza muscular y los niveles de Hidroxiprolina urinaria en jóvenes adultos. Mot. Hum., 12(2): 8-14, 2011.

\begin{abstract}
RESUMEN
El objetivo es investigar el efecto agudo del entrenamiento máximo de flexibilidad sobre fuerza muscular y los niveles de hidroxiprolina (HP) en adultos jóvenes. Treinta varones fueron divididos en grupo control $(G C, n=15 ; 16,87$ \pm 1,19 años) y grupo de entrenamiento máximo de flexibilidad (GF: No $=15,17,27 \pm 1,28$ años). Se midieron las concentraciones de HP a través del análisis de orina. La fuerza muscular se evaluó mediante un protocolo de dinamometría digital, con la ejecución de una repetición máxima de extensión de la columna lumbar (ECL). Después, el GF fue sometido a una sesión de entrenamiento máximo de flexibilidad, consistente de flexión del tronco en tres series con un tiempo de trabajo de 15 segundos. El GC no realizó ningún tipo de esfuerzo físico. Después de la sesión, se llevó a cabo una nueva evaluación de la fuerza muscular y un análisis de orina, realizados ambos 24 horas después de la primera. El ANOVA mostró reducción significativa en los valores de ECL $(? \%=-9,42, p=0,007)$ de GF, tras la sesión de flexión. En las comparaciones entre grupos, después de la intervención se encontraron valores en la fuerza muscular significativamente menores en el GF (?\%= -8,18, p =0,028), en comparación con el GC. En las concentraciones de HP no hubo cambios significativos. El protocolo de entrenamiento máximo de flexibilidad causó una reducción significativa en la fuerza muscular, sin embargo, no causó ninguna diferencia significativa en
\end{abstract} las concentraciones de HP.

Palabras clave: fuerza muscular, flexibilidad, hidroxiprolina.

\section{INTRODUCCIÓN}

Cuando se realizan trabajos físicos o actividades deportivas, es común la práctica de métodos para el desarrollo de la flexibilidad (1). Las técnicas más utilizadas para el entrenamiento de la flexibilidad son: estiramientos balísticos o dinámicos, estáticos y de facilitación neuromuscular propioceptiva (FNP) (2), y el empleo de las mismas está directamente relacionada con la intensidad $(3,4)$.

Las formas de entrenamiento de la flexibilidad se aplican en función del nivel de esfuerzo: el trabajo máximo y submáximo (estiramiento). El objetivo del entrenamiento máximo es mejorar los niveles de la flexibilidad, para lograr amplitudes del arco de movimiento mayores que las originales (5). Sin embargo, los ejercicios de estiramiento permiten el mantenimiento de un nivel adecuado de flexibilidad, con rango normal de movimiento, con la mínima restricción física posible y sin causar lesiones en el compartimento conectivo-múscular (6). Esta técnica puede reducir al mínimo el riesgo de lesiones en el tejido conectivo (7).

Los ejercicios de flexibilidad pueden ser perjudiciales para el desempeño de la fuerza cuando se aplican en la fase aguda, o sea, inmediatamente antes $(8,9,10,11,12,13)$, ya que pueden contribuir a la aparición de lesiones (14).

Algunas investigaciones $(15,16,17,18,19,20)$ afirman que el estiramiento, cuando se utiliza de forma submáxima, interfiere con el desempeño de la fuerza y si se aplica con la máxima intensidad puede reducir la capacidad de producción de fuerza y potencia. Silva (21) corrobora estos datos, indicando que las alteraciones e imposiciones 
ALMEIDA O.; MELLO D.; VALE R.; ROSA G.; SAMPAIO A.; DAOUD R.; LUQUE A. \& DANTAS E. Efecto agudo del entrenamiento máximo de flexibilidad sobre la fuerza muscular y los niveles de Hidroxiprolina urinaria en jóvenes adultos. Mot. Hum., 12(2): 8-14, 2011.

de la carga, pueden afectar a las estructuras musculares y conjuntivas que envuelven a los tendones, lo que interfiere con la producción de fuerza muscular.

Brown et al. (22) encontraron que el aumento en la generación de fuerza durante la contracción muscular, pueden afectar al metabolismo del colágeno así como a estructuras musculares y tendinosas del sistema músculo-esquelético. Por otra parte, otros estudios $(9,15,21,23,24,25,26,27)$ demuestran que existe una correlación positiva entre la capacidad de los estímulos para la flexibilidad y la fuerza y microlesiones del aparato locomotor, indicado por los mayores niveles de hidroxiprolina urinaria (HP).

Teniendo en cuenta los problemas presentados, este estudio tiene como objetivo examinar el efecto agudo del entrenamiento máximo de flexibilidad sobre la fuerza muscular y los niveles de HP en los adultos jóvenes.

\section{MATERIAL Y MÉTODO}

\section{Muestra}

La muestra fue obtenida de manera aleatoria entre los 500 estudiantes de la EPCAR y fue compuesta por 30 voluntarios varones, de edades comprendidas entre los 15 y los 20 años, y dividida en dos grupos: control (GC), y el de entrenamiento máximo de flexibilidad (GF). Se excluyeron los individuos que estaban fuera del grupo de edad indicado y los afectados por cualquier patología perceptible, declarada o detectada a través de un examen médico previo.

Todos los participantes fueron informados sobre los procedimientos de investigación, y en el caso de los niños menores de 18 años los tutores legales firmaron un formulario de consentimiento. El estudio fue aprobado por el Comité de Ética en la Investigación de la Universidad Castelo Branco (UCB - RJ), en virtud del Protocolo. 0094/2008, de acuerdo con las normas para la realización de investigaciones en seres humanos del Consejo Nacional de Salud, la Resolución 196/96 del 10/10/96, y la Declaración de Helsinki (28).

\section{Procedimientos para la recogida de datos}

Con el objetivo de caracterizar la muestra, se obtuvieron las medidas antropométricas de la masa corporal y la altura, y el porcentaje de grasa corporal por tres pliegues cutáneos (pectoral, abdominal y muslo anterior) tal y como recoge el protocolo descrito por Jackson y Pollock (29). Todas las mediciones fueron realizadas por profesionales entrenados para este propósito.
Para la evaluación de la masa corporal, se utilizó una balanza digital PL150 con capacidad de $150 \mathrm{~kg}$ y precisión de 100 g de Filizola ${ }^{\circledR}$ (Brasil). La medición de la talla se realizó con un estadiómetro modelo profesional Sanny ® (Brasil). Para recoger los valores de los pliegues cutáneos se utilizó un plicómetro o lipocalibre Lange® profesional, con $1 \mathrm{~mm}$ de precisión y la presión constante de $10 \mathrm{~g} / \mathrm{mm} 2$.

En todas las medidas antropométricas se adoptaron los procedimientos establecidos por la International Society for the Advancement of Kinanthropometry - ISAK (30). Para evaluar los daños producidos en el colágeno muscular se utilizó como marcador bioquímico la hidroxiprolina (HP), cuya concentración fue medida en orina. La recogida de la muestra se realizó el día después de la evaluación antropométrica.

Los participantes recibieron instrucciones para no ingerir colágeno presente en algunos alimentos (carnes rojas y blancas, mariscos, dulces, helados o jalea) dos días antes de la prueba. Las muestras de orina fueron recogidas siguiendo las directrices del método Nordin (31), en los que durante el período de 2 horas (de 7 a 9 horas), después de que los sujetos fueran sometidos a 12 horas de ayuno, se recomendó la ingesta de agua antes de la colecta. Las botellas de un litro fueron identificadas correctamente por el laboratorio São Lucas, Barcbacena/ MG/Brasil, cuya certificación de la calidad tiene por objeto social la norma ISO 9001/2000.

Para determinar las concentraciones urinarias de HP, se utiliza el protocolo HPROLI $2 \mathrm{~h}$ método colorimétrico (31). Las muestras fueron analizadas en el sistema HPLC, que consiste en una bomba de gradiente, una válvula de inyección, una columna de calor $\left(60^{\circ} \mathrm{C}\right)$, un detector de UV / VIS 472nm a un ordenador con la HPLC y un pulso regulador Este proceso de captura se lleva a cabo por una célula fotoeléctrica, se amplifica electrónicamente y se registra por la computadora el pico proporcional a la concentración de HP. Tras el análisis de orina, los valores obtenidos de HP son convertidos de mg/l a $\mathrm{mg} / 24 \mathrm{~h}$, para permitir una comparación directa con los valores de referencia.

La evaluación de la fuerza muscular se realizó en la tarde, con el uso del protocolo de dinamometría digital, mediante la ejecución de una repetición máxima (1RM) (32), del movimiento de la extensión de la columna lumbar (ECL). La medición se realizó con un dinamómetro digital de usos múltiples, una cadena CEFISA $®$ (Brasil) con la célula de carga para PC, resolución de $0,1 \mathrm{kgf} 1 \mathrm{~N}$ o la capacidad máxima de 250 kgf o $2500 \mathrm{~N}$ acoplado a un sistema de adquisición de datos N2000. 
Inmediatamente después de la evaluación de la fuerza muscular se aplicó el programa de ejercicios de flexión en el GF: flexión del tronco por parejas en la posición sentada en tres series, con una duración de 15 segundos, forzando hasta el límite articular con el fin de lograr el mayor rango de movimiento posible. Para controlar la intensidad, se tomó como base la escala de esfuerzo percibido en la flexibilidad (Perflex) (1).

Se observó que la percepción del esfuerzo en el GF se mantuvo en el rango de malestar, entre 61 y 80 de la misma escala, el valor actual de $65,7 \pm 3,7$. El GC permaneció en el lugar de intervención, sin realizar ningún esfuerzo físico.

Una nueva evaluación de la fuerza muscular se realizó inmediatamente después de la intervención, con el objetivo de comparar los datos. Con el mismo objetivo, la colectas de muestras de orina post-test fueron tomadas 24 horas después de la recogida de orina pretest.

\section{Análisis estadístico}

Los datos fueron procesados por el paquete estadístico SPSS 18 para Windows y se presentan los valores medios, desviación estándar, máximos, mínimos y las diferencias de porcentaje (?\%). Los preocedimientos estadísticos Shapiro-Wilk y Levene se aplicaron para comprobar la normalidad y la homogeneidad de varianza de la muestra, respectivamente. Empleamos el análisis de varianza con medidas repetidas en el grupo de factores (GC, GF) y el tiempo (pre y post-test), seguido por el test post hoc para identificar las posibles diferencias dentro y entre los grupos. El estudio estableció el nivel de $p<0,05$ para la significación estadística.

\section{RESULTADOS}

La Tabla 1 presenta las características antropométricas de los individuos en los grupos seleccionados, y los resultados de la prueba de Shapiro-Wilk (SW) para evaluar la normalidad de la muestra. Se observa que todas las variables tienen valores cerca de la curva normal.

\begin{tabular}{|c|c|c|c|c|}
\hline & Edad (años) & MC (Kg) & Est. (m) & $\% \mathbf{G}$ \\
\hline GC & $16,87 \pm 1,19$ & $63,04 \pm 5,92$ & $171,60 \pm 5,54$ & $10,55 \pm 3,29$ \\
\hline p-valor (SW) & 0,279 & 0,554 & 0,354 & 0,477 \\
\hline GF & $17,27 \pm 1,28$ & $63,72 \pm 5,29$ & $175,20 \pm 4,14$ & $9,47 \pm 3,63$ \\
\hline p-valor (SW) & 0,094 & 0,997 & 0,162 & 0,095 \\
\hline
\end{tabular}

La figura 1 presenta los datos sobre la fuerza muscular pre-y post-intervención en el GC y GF. Hubo una reducción significativa en los valores de ECL $(? \%=-9,42, p$ $=0,007)$ en el GF, tras la sesión de flexión. En las comparaciones entre grupos, después de la intervención, hubo una reducción en los valores del GF $(? \%=-8,18, p$ $=0,028)$, en comparación con el GC.

La figura 2 muestra los datos de HP en orina pre y postintervención en el GC y GF. Se puede ver que a pesar de la tendencia a la disminución en las concentraciones de servaron diferencias estadísticamente significativas intra e inter grupos.

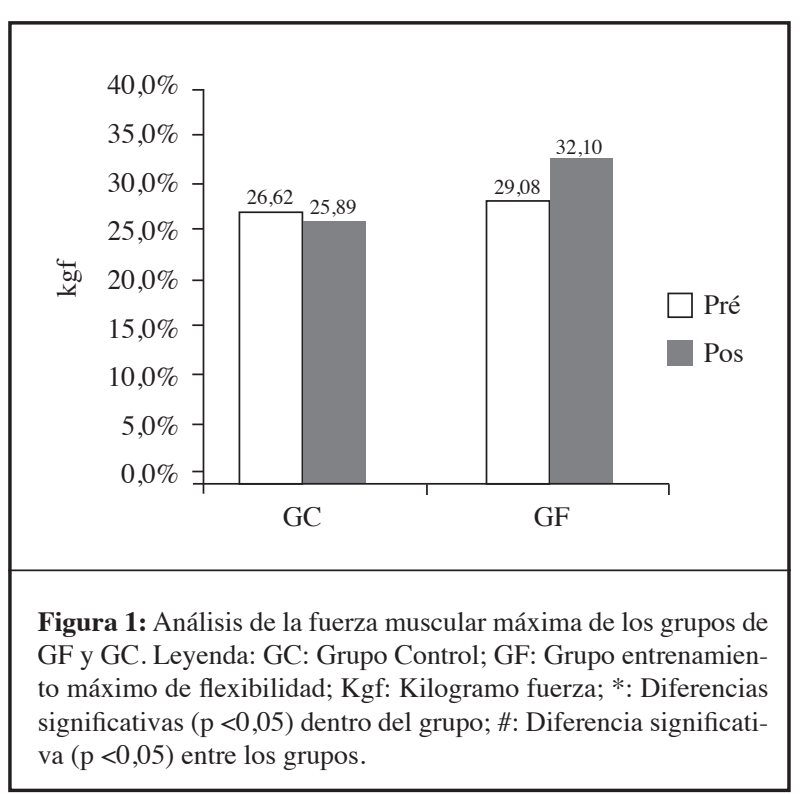


ALMEIDA O.; MELLO D.; VALE R.; ROSA G.; SAMPAIO A.; DAOUD R.; LUQUE A. \& DANTAS E. Efecto agudo del entrenamiento máximo de flexibilidad sobre la fuerza muscular y los niveles de Hidroxiprolina urinaria en jóvenes adultos. Mot. Hum., 12(2): 8-14, 2011.

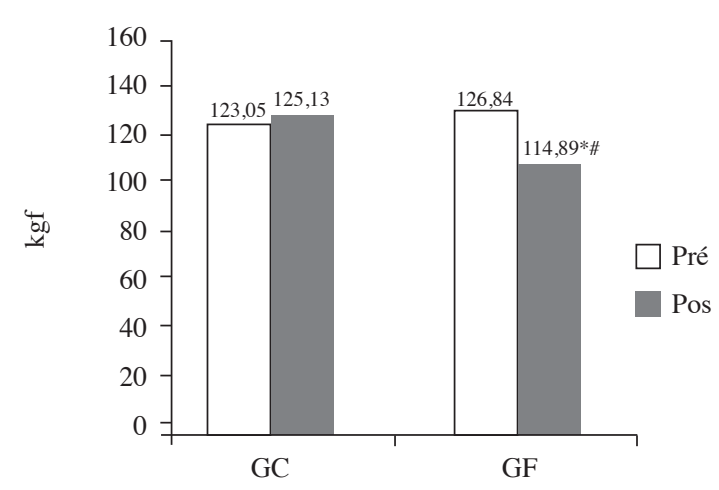

Figura 2: Análisis de los niveles urinarios de HP y los grupos GC y GF. Leyenda: GC: Grupo Control; GF: Grupo entrenamiento máximo de flexibilidad; *: Diferencias significativas $(\mathrm{p}<0,05)$ dentro del grupo; \#: Diferencia significativa $(\mathrm{p}<0,05)$ entre los grupos.

\section{DISCUSIÓN}

Los resultados de este estudio demuestran que el programa de entrenamiento máximo de flexibilidad causó una reducción en la fuerza muscular de los sujetos en el GF. Estos datos son consistentes con los resultados de la Conceição et al. (20), Cardozo et al. (33), y Galdino et al. (34), quienes llegaron a la conclusión de que el ejercicio permitió duplicar las pérdidas en la fuerza muscular después de la aplicación del método estático.

Los resultados de la investigación de Power et al. (10) son similares a los del presente estudio, en los que también se observó una disminución de la fuerza muscular de los sujetos después del entrenamiento máximo de flexibilidad.

Según Endlich et al. (15), la reducción de fuerza muscular puede estar relacionada con los mecanismos neuronales. Cramer et al. (14) informaron que la intervención aguda puede actuar en los órganos tendinosos de Golgi, provocando la liberación de neurotransmisor inhibitorio ácido gamma-aminobutírico en la médula ósea, dando lugar a disminución de la fuerza muscular.

Para Prati et al. (9), la reducción de la fuerza se debe a un cambio en las propiedades mecánicas de los músculos relacionado con la tensión y la longitud, mientras que Behm et al. (35) informó que la reducción de la fuerza está más afectada por la inhibición de los músculos que por el cambio en la elasticidad de los mismos. Estas hipótesis apoyan los datos obtenidos en este estudio, a través del cual se puede sugerir que la disminución de la fuerza máxima de la muestra se debe a los factores descritos anteriormente, provocados por el estímulo del aumento de la amplitud máxima de movimiento.

En cuanto a las concentraciones de HP, Nascimento et al. (36) observaron un aumento significativo de esta variable después de la práctica del entrenamiento máximo de flexibilidad en medio acuático y terrestre en adultos no atletas. Estos datos difieren de los obtenidos en este estudio, sin embargo, las diferencias entre los protocolos de intervención utilizados pueden explicar la discrepancia entre los resultados.

Las investigaciones realizadas por Virtanen et al. (37) demostraron que el ejercicio concéntrico no provocaría un aumento de los niveles urinarios de HP. Estos datos son similares a los del presente estudio sobre la respuesta de las concentraciones de HP.

Murguía et al. (38) observaron altos niveles de HP en pacientes sometidos a ejercicios de flexión, que muestran aparición de lesiones en el tejido. A pesar de que difieren de las conclusiones de este estudio, estos datos son consistentes con los obtenidos por Rocha-Mafra et al. (39), quienes observaron un aumento significativo en los niveles de HP de las mismas personas después de someterse a una sesión de flexión estática.

En el estudio realizado por Silva et al. (21) no se observaron cambios significativos en la fase aguda de los niveles de HP en individuos sometidos a ejercicios de estiramiento. El presente estudio obtuvo resultados similares a estos, lo que corrobora la hipótesis de que la intensidad puede ser la variable clave para los cambios en las concentraciones de HP.

Chaves et al. (40) sostienen en su estudio que los cambios en las capacidades físicas como la flexibilidad, pueden estar sujetos a una considerable variación individual en las mujeres debido a las diferentes fases del ciclo menstrual. Como los participantes de este estudio eran de sexo masculino, se necesitan más investigaciones que puedan abordar la relación entre el entrenamiento de la flexibilidad y los niveles de HP en las mujeres.

El protocolo de entrenamiento máximo de flexibilidad que se utiliza en este estudio fue capaz de causar una reducción significativa en la fuerza muscular, sin embargo, no causó ninguna diferencia significativa en las concentraciones de HP.

Los resultados sugieren que los ejercicios de entrenamiento máximo de flexibilidad no deben aplicarse como estímulo anterior en actividades que requieran 
máxima potencia, aunque se no se detectaron microlesiones determinadas por la no alteración de los niveles de HP en la orina.

En otras investigaciones futuras se recomienda que se analicen los efectos agudos y crónicos de las variables estudiadas, así como la aplicación en personas de diferentes edades, sexo y niveles de condición física.

\section{REFERENCIAS BIBLIOGRÁFICAS}

1. Dantas EHM, Salomão PT, Vale RGS, Achour Júnior A, Simão R, Figueiredo NMA. Escala de esfuerzo percibido en la flexibilidad (PERFLEX): ¿un instrumento adimensional para evaluarse la intensidad? Fit Perf J. 2008;7(5):289-94.

2. Nogueira CJ, dos Santos Galdino LA, Souza Valle RG, Martin Dantas EH. Efeito agudo do alongamento submáximo e do método de Facilitação Neuromuscular Proprioceptiva sobre a força explosiva. HU Revista. 2009;35(1).

3. Kraemer WJ, Ratamess NA. Fundamentals of resistance training: progression and exercise prescription. Med Sci Sports Exerc. 2004;36(4):674.

4. Tan B. Manipulating resistance training program variables to optimize maximum strength in men: a review. J Strength Cond Res. 1999;13:289-304.

5. Viveiros L, Polito MD, Simão R, Farinatti P. Respostas agudas imediatas e tardias da flexibilidade na extensão do ombro em relação ao número de séries e duração do alongamento. Rev Bras Med Esporte 2004;10:459-63.

6. Azevedo DC, Carvalho SCd, Leal EWPS, Damasceno SP, Ferreira ML. Influência da limitação da amplitude de movimento sobre a melhora da flexibilidade do ombro após um treino de seis semanas. Rev Bras Med Esporte 2008;14:119-21.

7. Grego Neto A, Manffra EF. Influência do volume de alongamento estático dos músculos isquiotibiais nas variavéis isocinéticas. Rev Bras Med Esporte:104-9.

8. Nelson AG, Allen JD, Cornwell A, Kokkonen J. Inhibition of maximal voluntary isometric torque production by acute stretching is joint-angle specific. Res Q Exerc Sport. 2001;72(1):68.

9. Prati J, Carvalho Machado SE, Sobrinho AHJ, Alencar Carvalho MCG, Dantas EHM. O efeito agudo do flexionamento passivo sobre a força máxima: um estudo experimental. Fit Perf J. 2006;5(5):311-7.

10. Power K, Behm D, Cahill F, Carroll M, Young W. An acute bout of static stretching: effects on force and jumping performance. Med Sci Sports Exerc. 2004;36(8):1389.

11. Wallmann HW, Mercer JA, MCwhorter J. Surface electromyographic assessment of the effect of static stretching of the gastrocnemius on vertical jump performance. J Strength Cond Res. 2005;19(3):684.

12. Paiva Carvalho FL, Prati JELR, Alencar Carvalho MCG, Dantas EHM. Efeitos agudos do alongamento estático e da facilitação neuromuscular proprioceptiva no desempenho do salto vertical de tenistas adolescentes. Fit Perf J. 2009;8(4):264-8.

13. Magnusson S, Simonsen E, Aagaard P, Moritz U, Kjaer M. Contraction specific changes in passive torque in human skeletal muscle. Acta Physiol Scand. 1995;155(4):377-86.

14. Cramer JT, Housh TJ, Johnson GO, Miller JM, Coburn JW, Beck TW. Acute effects of static stretching on peak torque in women. J Strength Cond Res. 2004;18(2):236

15. Endlich PW, Farina GR, Dambroz C, Gonçalves WLS, Moysés MR, Mill JG, et al. Efeitos agudos do alongamento estático no desempenho da força dinâmica em homens jovens. Rev Bras Med Esporte 2009;15(3):200-3.

16. Fowles J, Sale D, MacDougall J. Reduced strength after passive stretch of the human plantarflexors. J Appl Physiol. 2000;89(3):1179.

17. Arruda FB, Faria LB, Silva W, Simão R, Senna GW, Novaes J. A influência do alongamento no rendimento do treinamento de força. Rev Trein Desp 2006;7:1-5.

18. Cornwell A, Nelson A, Heise G, Sidaway B. Acute effects of passive muscle stretching on vertical jump performance. J Human Mov Studies. 2001;40(4):307.

19. Young W, Behm D. Effects of running, static stretching and practice jumps on explosive force production and jumping performance. J Sports Med Phys Fitness. 2003;43(1):21-7. 
ALMEIDA O.; MELLO D.; VALE R.; ROSA G.; SAMPAIO A.; DAOUD R.; LUQUE A. \& DANTAS E. Efecto agudo del entrenamiento máximo de flexibilidad sobre la fuerza muscular y los niveles de Hidroxiprolina urinaria en jóvenes adultos. Mot. Hum., 12(2): 8-14, 2011.

20. Conceição MCSC, Vale RGS, Bottaro M, Dantas EHM, Novaes JS. Efeitos de quatro tempos diferentes de permanência de flexionamento estático na flexibilidade de adultos jovens. Fit Perf J. 2008;7(2):88-92.

21. Silva KLGL, Coelho RAP, Marins JCB, Dantas EHM. Efeitos do alongamento sobre os níveis de hidroxiprolina em atiradores do tiro de guerra. Fit Perf J. 2005(6):348.

22. Brown SJ, Child RB, Day SH, Donnelly AE. Indices of skeletal muscle damage and connective tissue breakdown following eccentric muscle contractions. Eur J Appl Physiol Occup Physiol. 1997;75(4):369-74.

23. Nascimento V, Oliveira C, Junior H, Dantas E. Níveis de HP em Adultos Submetidos ao Flexionamento Dinâmico nos Meios Líquidos e Terrestre. Fit Perf J. 2005; 3: 150-156.

24. Caetano L, Mesquita M, Lopes R, Pernambuco C, Silva E, Dantas E. Hidrocinesioterapia na redução lombar avaliada através dos níveis de hidrpxiprolina e dor. Fit Perf J. 2005;5(1):38-42.

25. Mesquita MG, Caetano L, Pernambuco CS, Silva EB, Dantas EHM. Análise da excreção urinária de hidroxiprolina e do nível de dor em sujeitos com lombalgia após alongamento na hidrocinesioterapia. Fisioter Bras 2007;8(2):99-106.

26. Nogueira A, Simao R, Carvalho M, Vale R, Dantas E. Concentracao de hidroxiprolina como marcador bioquimico do dano musculo esquetico apos treinamento de resistencia de forca. R Bras Ci e Mov. 2007;15(2):33-8.

27. Pernambuco CS, Laranjeira C, Mesquita MG, Conceição MCSC, Vale RGS, Dantas EHM. Urinary concentration of hidroxiproline, on men with low back pain submited to hydrokinesiotherapy. Mot Hum 2010;11(2):80-6.

28. W.M.A. Declaration of Helsinki. Ethical Principles for Medical Research Involving Human Subjects. 59th WMA General Assembly, Seoul 2008.

29. Jackson A, Pollock M. Generalized equations for predicting body density of men. Br J Nutr. 2007;40(03):497-504.

30. Marfell-Jones M. International standards for anthropometric assessment. ISAK: Potchefsroom, South Africa 2006.

31. Nordin B, Hodgkinson A, Peacock M. The measurement and the meaning of urinary calcium. Clin Orthop Relat Res. 1967;52:239-322.

32. Marinho PC, Junior OA. Mensuração da força isométrica e sua relação com a velocidade máxima de jovens nadadores com diferentes níveis de performance. R Bras Ci e Mov. 2008;12(1):71.

33. Cardozo G, Torres JB, Dantas EHM, Simão R. Comportamento da Força Muscular após o Alongamento Estático. Rev Trein Desp 2006;7(1):73-6.

34. Galdino LAS, Nogueira CJ, César EP, Fortes MEP, Perrout JR, Dantas EHM. Comparação Entre Níveis de Força Explosiva de Membros Inferiores Antes e Após Flexionamento Passivo. Fit Perf J. 2005;4(1):11-5.

35. Behm DG, Button DC, Butt JC. Factors affecting force loss with prolonged stretching. Appl Physiol Nutr Metab. 2001;26(3):262-72.

36. Nascimento V, Silva K, Caetano L, Mesquita M, Nogueira A, Lopes R, et al. Nível de lesão, avaliada pela hidroxiprolina, produzida pelo flexionamento dinâmico no meio líquido. Rev Mineira Educ Fís. 2004;12(2):596.

37. Virtanen P, Viitasalo J, Vuori J, Vaananen K, Takala T. Effect of concentric exercise on serum muscle and collagen markers. J Appl Physiol. $1993 ; 75(3): 1272$.

38. Murguia M, Vailas A, Mandelbaum B, Norton J, Hodgdon J, Goforth H, et al. Elevated plasma hydroxyproline. Am J Sports Med. 1988;16(6):660.

39. Rocha Mafra O, da Silva E, Giani T, Neves C, Lopes R, Dantas E. Hydroxyproline Levels in Young Adults Undergoing Muscular Stretching and Neural Mobilization. J Med Biochem. 2010;29(1):39-43.

40. Chaves C, Simão R, Araújo C. Ausência de variação da flexibilidade durante o ciclo menstrual em universitárias. Rev Bras Med Esporte $2002 ; 8(6)$. 


\begin{abstract}
The aim was to investigate the acute effect of maximal stretching training on muscular strength and hydroxyproline levels (HP) in young adults. Thirty men were shared in: control group (GC: $n=15 ; 16,87 \pm 1,19$ years old) and maximal stretching training group (GF: $n=15 ; 17,27 \pm 1,28$ years old). HP concentrations were assessed. Muscular strength was evaluated through digital dynamometry protocol, with a perform of one maximal repetition of low back extention movement (ECL). Immediately after the muscle strength evaluation, the GF was undergone to a stretching session: three sets, with insistence time of 15 seconds of trunk flexion movement. The GC did not realize any kind of physical effort. After the intervention, new evaluation of muscular strength was realized, and the new urine collection was made 24 hours after the first sample collection. The ANOVA showed a significant reduction on ECL values (?\%= $-9,42 ; \mathrm{p}=0,007)$ of GF after the stretching session. In the intergroups comparation, at the post intervention moment, significant lower muscular strength values $(? \%=-8,18 ; \mathrm{p}=0,028)$ on GF were observed in relation to GC. The HP concentrations did not present significant alterations. The maximal stretching training protocol promoted significant reduction in muscular strength, however, did not promote significant alterations on HP concentrations.
\end{abstract}

Keywords: Muscular Strength, Hydroxyproline, Flexibility.

\title{
Dirigir correspondencia a:
}

Guilherme Rosa

Rua Piraquara, 879, BL2, Apto508. Realengo.

Rio de Janeiro - RJ. Brasil.

CEP: $21755-270$

Fone: 5521 7833-2270 / 5521 2401-5243

grfitness@hotmail.com

RECIBIDO: 03-10-2011

ACEPTADO: 13-12-2011 